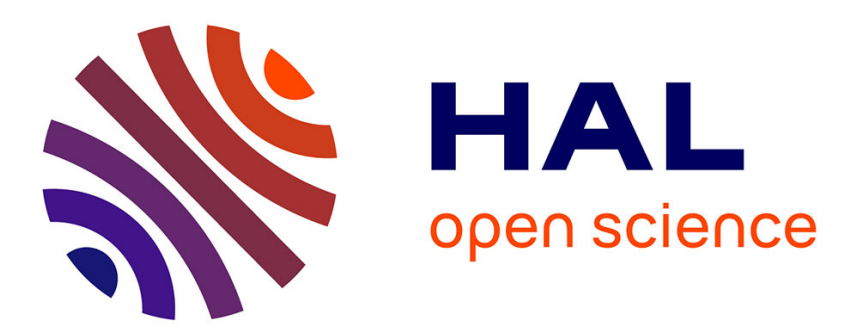

\title{
Les corvées domestiques sont-elles solubles dans le jeu vidéo?
}

Fanny Lignon

\section{To cite this version:}

Fanny Lignon. Les corvées domestiques sont-elles solubles dans le jeu vidéo?. Sociétés \& Représentations, 2018, Représentation et non-représentation des Roms en Espagne et en France, 45, pp.157-171. 10.3917/sr.045.0157 . hal-01802067

\section{HAL Id: hal-01802067 https://hal.science/hal-01802067}

Submitted on 28 May 2018

HAL is a multi-disciplinary open access archive for the deposit and dissemination of scientific research documents, whether they are published or not. The documents may come from teaching and research institutions in France or abroad, or from public or private research centers.
L'archive ouverte pluridisciplinaire HAL, est destinée au dépôt et à la diffusion de documents scientifiques de niveau recherche, publiés ou non, émanant des établissements d'enseignement et de recherche français ou étrangers, des laboratoires publics ou privés. 


\section{Les corvées domestiques sont-elles solubles dans le jeu vidéo?}

Ménage, cuisine, repassage... Faire la liste des " corvées domestiques » semble aller de soi. Mais délimiter avec exactitude ce qui relève des " activités relatives à l'entretien de l'espace résidentiel et de ses membres ${ }^{1}$ " est cependant loin d'être évident. En France, l'Institut national de la statistique et des études économiques (INSEE), pour effectuer ses enquêtes, retient ordinairement trois critères. Pour entrer dans la catégorie "travail domestique ", une tâche doit être productive, non rémunérée, susceptible d'être déléguée (contre rémunération) à quelqu'un d'autre. Trois périmètres sont distingués :

- le périmètre restreint, qui regroupe le cœur des tâches domestiques (cuisine, ménage, soins matériels aux enfants, entretien du linge, gestion du ménage) et auquel les foyers ont consacré, en 2012, si l'on se réfère aux chiffres publiés ${ }^{2}, 2$ h 07 de temps par jour;

- le périmètre intermédiaire, qui reprend la liste précédente en y ajoutant des activités qui se situent à la frontière des loisirs : courses, jardinage, bricolage, jeux avec les enfants (3 heures par jour);

- le périmètre large, qui tient compte de l'ensemble des tâches citées et de quelques autres encore : promener un animal, effectuer un trajet en voiture pour se déplacer soi-même (4 heures par jour).

Il apparaît qu'en 25 ans, le temps quotidien consacré aux tâches domestiques a diminué de 30 minutes. Cette baisse s'explique par la réduction 
massive du temps passé au noyau dur des activités domestiques, mais ne s'observe que du côté des femmes. Du côté des hommes en effet, le temps consacré à ces tâches n'a pas évolué. Ce qui revient à dire que les femmes en font moins, mais que les hommes n'en font pas plus. Aussi, plus on se rapproche du cœur du problème, plus la part réalisée par les femmes est importante : $60 \%$ pour le périmètre le plus large, $72 \%$ pour le périmètre le plus restreint ${ }^{3}$. En conclusion, s'il apparaît que l'inégalité entre les sexes face aux tâches domestiques tend à se réduire, elle continue toutefois de persister.

Agir sur un plan éducatif pour faire évoluer cette situation est l'une des pistes actuellement explorée. La Convention interministérielle pour l'égalité entre les filles et les garçons, les femmes et les hommes dans le système éducatif 2013$2018^{4}$ enjoint aux enseignants des écoles, des collèges, des lycées et du supérieur :

- d'acquérir et transmettre une culture de l'égalité entre les sexes;

- de renforcer l'éducation au respect mutuel et à l'égalité entre les filles et les garçons, les femmes et les hommes;

- de s'engager pour une plus grande mixité des filières de formation à tous les niveaux d'étude.

Le Socle commun de connaissances, de compétences et de culture ${ }^{5}$, qui concerne les élèves de 6 à 16 ans, inscrit ces exigences dans le chapitre consacré à la formation de la personne et du citoyen, précisant que ce domaine fait appel « à l'apprentissage et à l'expérience des principes qui garantissent la liberté de tous, comme la liberté de conscience et d'expression, la tolérance réciproque, l'égalité, notamment entre les hommes et les femmes, le refus des discriminations, l'affirmation de la capacité à juger et agir par soi-même. " Dans le paragraphe "expression de la sensibilité et des opinions, respect des autres ", au rang des objectifs, il est écrit :

Apprenant à mettre à distance préjugés et stéréotypes, il [l'élève] est capable d'apprécier les personnes qui sont différentes de lui et de vivre avec elles.

Par ailleurs, on peut lire sur le site du ministère de l'Éducation nationale, de l'enseignement supérieur et de la recherche que «la première pratique culturelle des jeunes est celle de l’image : cinéma, photo, télévision, jeux vidéo,

3. Il s'agit bien évidemment de moyennes, les chiffres variant selon l'âge des personnes, la situation familiale, le nombre d'enfants à charge.

4. En ligne: http://www.education.gouv.fr/pid25535/bulletin_officiel.html?cid_bo=67018, consulté le 19 juillet 2017].

5. En ligne: http://eduscol.education.fr/cid86943/le-socle-commun.html-lien0, consulté le juillet 2017. Décret n² 2015-372 du 31-3-2015 - J.O. du 2-4-2015. 
Internet, etc. ", et que «l'école donne une place importante au cinéma et à l'audiovisuel dans le cadre plus large de l'éducation à l'image ${ }^{6}$.

Pourquoi, dès lors, ne pas joindre ces demandes - éduquer simultanément à l'image et à l'égalité femmes/hommes - et prendre comme support un jeu vidéo?

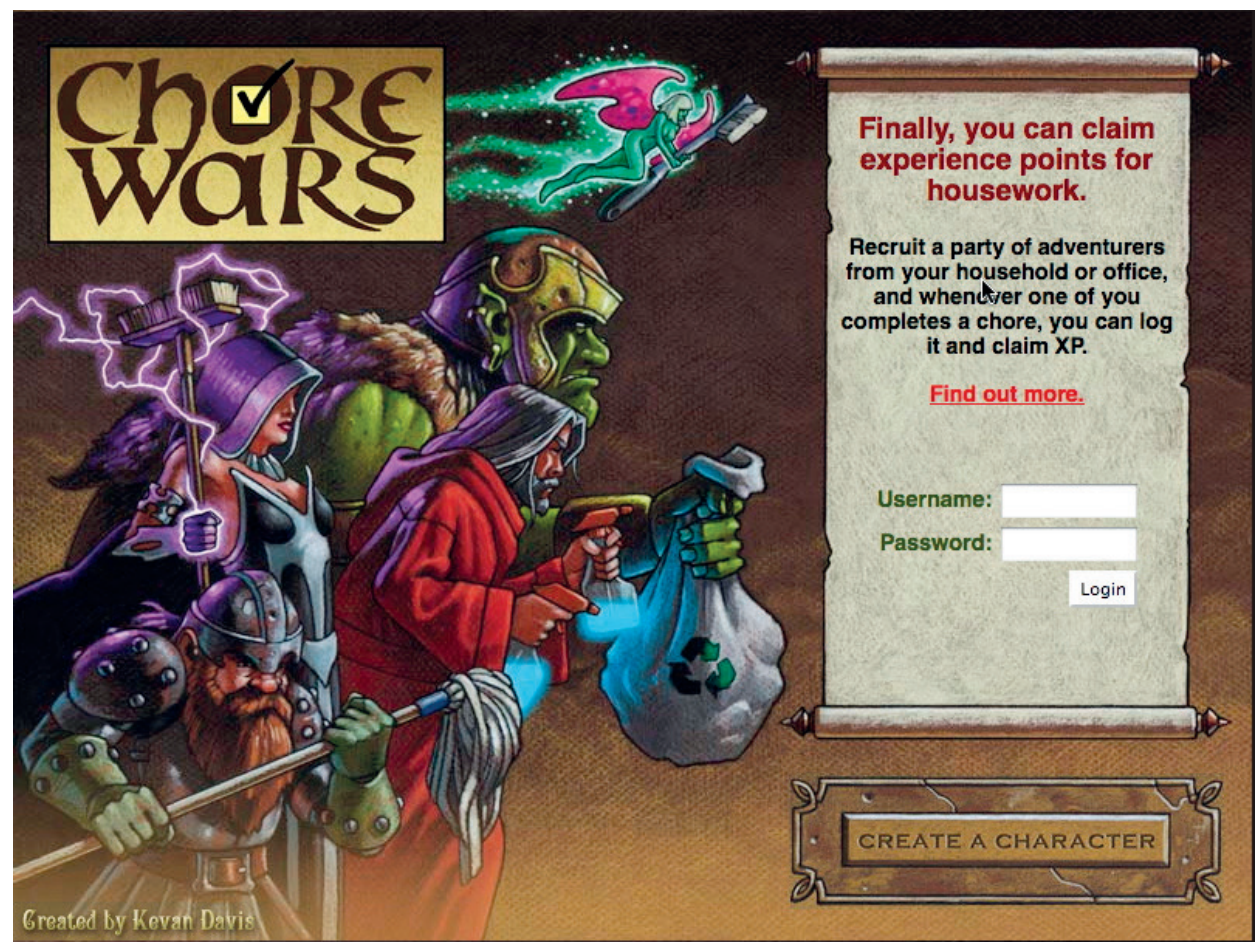

Ill. 1 - Chore Wars. Page d'accueil

\section{Chore Wars : principe et modalités}

Chore Wars ("Les guerres des corvées») a été imaginé en 2007 par Kevan Davis, un développeur et game designer anglais. Sur sa page personnelle, il présente le jeu en ces termes :

A chore management system where a household, family or office can create a party of adventurers, who are then able to claim experience points for completing chores?

6. En ligne : http://www.education.gouv.fr/cid21004/l-education-a-l-image-au-cinema-et-a-l-audiovisuel.html, consulté le 19 juillet 2017.

7. En ligne : http://kevan.org/cv, consulté le $1^{\text {er }}$ septembre 2017 : « Outil de gestion des corvées. La possibilité pour un ménage, une famille, des collègues de bureau, de créer un groupe d'aventuriers qui vont pouvoir gagner des points d'expérience en accomplissant des corvées. » (Traduction de l'auteure.)

Fanny Lignon, "Les corvées domestiques sont-elles solubles dans le jeu vidéo? », S. \& R., n 45, printemps 2018, p. 157-171. 
Sur sa page LinkedIn, il ajoute: Web service for tracking household or workplace chores within a frivolous dungeons-and-monsters framework ${ }^{8}$. Si le programme permet de mesurer la part de travail domestique effectuée par chacun, il n'impose rien cependant quant à l'usage qui peut être fait des données recueillies. L'analyse des témoignages de joueurs publiés sur le site du jeu porte néanmoins à notre connaissance trois modalités d'utilisation approuvées par l'auteur :

Gérer :

Le jeu consiste en une liste de corvées qui doivent être effectuées.

Il permet de s'assurer de leur réalisation, de leur répartition sur une période donnée, de l'équité de cette répartition. Il permet aussi de suivre une corvée en particulier (qui l'effectue, quand, à quelle fréquence?) ${ }^{10}$;

Récompenser :

De façon symbolique, en donnant un prix à celle ou celui qui aura fait le meilleur score sur une période donnée, de façon concrète, en convertissant les gains virtuels en avantages, privilèges ou cadeaux pour les participants;

\section{Sanctionner :}

En imposant à celle ou celui qui en a fait le moins d'effectuer telle ou telle corvée urgente ou spécifique (principe du gage).

La page d'accueil ${ }^{11}$ présente cinq héros : un orque, un mage, un nain, une sorcière et une fée. Les personnages masculins sont plus nombreux que les personnages féminins et occupent plus d'espace. Ils sont au premier plan et en première ligne. La sorcière cependant, si elle se tient légèrement en retrait ${ }^{12}$, est le pivot du groupe. En témoignent sa posture, très droite, très affirmée, et l'aura violette qui émane de sa personne et projette une lumière colorée sur les hommes qui l'entourent. Quant à la fée poussière, elle a beau être de petite

8. En ligne : https://www.linkedin.com/in/kevandavis, consulté le 1er septembre 2017 : « Service Web pour le suivi des tâches ménagères, à la maison ou au bureau, dans une ambiance fantaisiste de type "donjons et dragons". » (Traduction de l'auteure.)

9. En ligne: http://www.chorewars.com/testimonials.php, consulté le 1er septembre 2017. Certes, aucun élément ne permet d'évaluer le degré d'authenticité des témoignages figurant sur cette page, mais les propos publiés reflètent nécessairement des pratiques et usages voulus par l'auteur du jeu et/ou qui lui conviennent.

10. L'un des joueurs qui témoigne déclare souffrir de troubles de la mémoire et explique qu'il se sert du jeu pour pallier son handicap.

11. En ligne : http://www.chorewars.com/, consulté le 1er septembre 2017.

12. Ce qui, par ailleurs, correspond à son rôle de jeteuse de sorts.

Fanny Lignon, « Les corvées domestiques sont-elles solubles dans le jeu vidéo? », S. \& R., nº 45, printemps 2018, p. 157-171. 
taille, elle n'en mène pas moins l'assaut ${ }^{13}$. En lieu et place des armes attendues, les personnages brandissent des ustensiles de nettoyage.

Deux univers distincts sont donc ici mis en scène: l'univers ménager et l'univers de l'Heroic Fantasy. Le visuel en effet évoque les MMORPG ${ }^{14}$, et notamment World of Warcraft, tant au niveau de la charte graphique que des costumes et attitudes des héros. Cette page d'accueil donne ainsi d'emblée le ton, l'humour se construisant sur l'association incongrue d'éléments relevant de deux domaines qui n'ont d'ordinaire rien à voir l'un avec l'autre. Le balai espagnol joue les rapières, les vaporisateurs d'Ajax Vitres les fioles de mana, et les pièces d'armures les tabliers et autres gants de vaisselle; le thème de la guerre assurant leur jonction. Le discours véhiculé par cette image est clair. Tous unis contre un ennemi commun : la poussière.

Les ressorts ludiques sur lesquels est fondé Chore Wars sont assez classiques : la compétition, l'obtention de récompenses symboliques, le hasard. Le jeu s'inspire des jeux de rôles sur table et en ligne. On y trouve des personnages, des aventures, des quêtes, des combats. Lors de la phase de création des personnages, le joueur choisit un avatar parmi ceux que lui propose l'ordinateur.
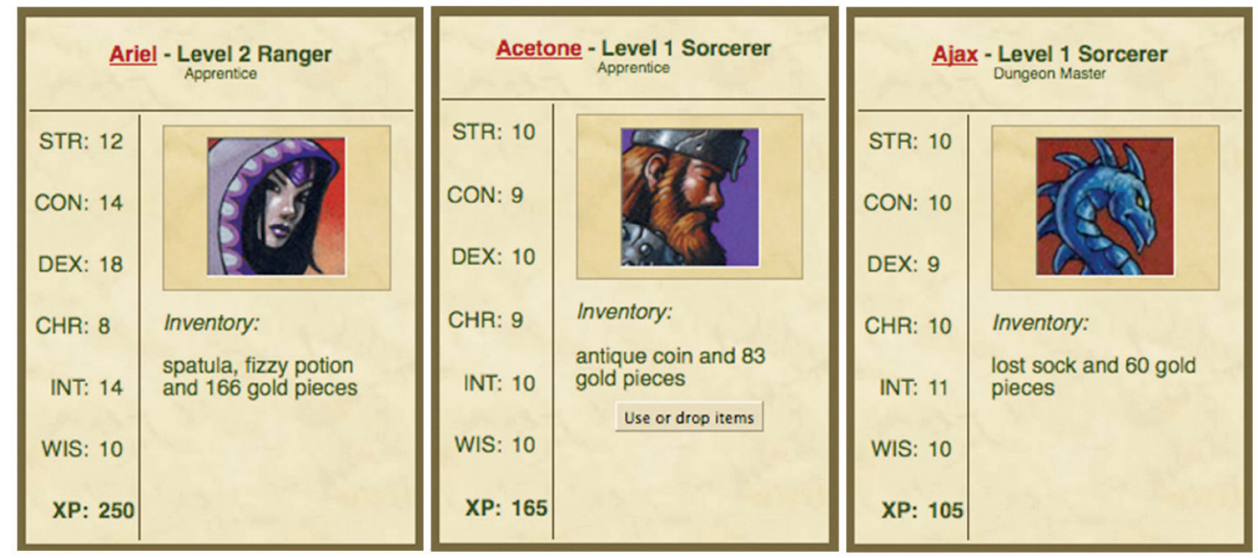

Ill. 4 - Fiches personnages : trois exemples (capture d'écran)

13. On notera la dynamique gauche/droite à l'œuvre dans l'image.

14. Massively Multiplayer Online Role-Playing Game : jeu de rôle en ligne massivement multijoueur.

Fanny Lignon, "Les corvées domestiques sont-elles solubles dans le jeu vidéo? », S. \& R., n 45, printemps 2018, p. 157-171. 


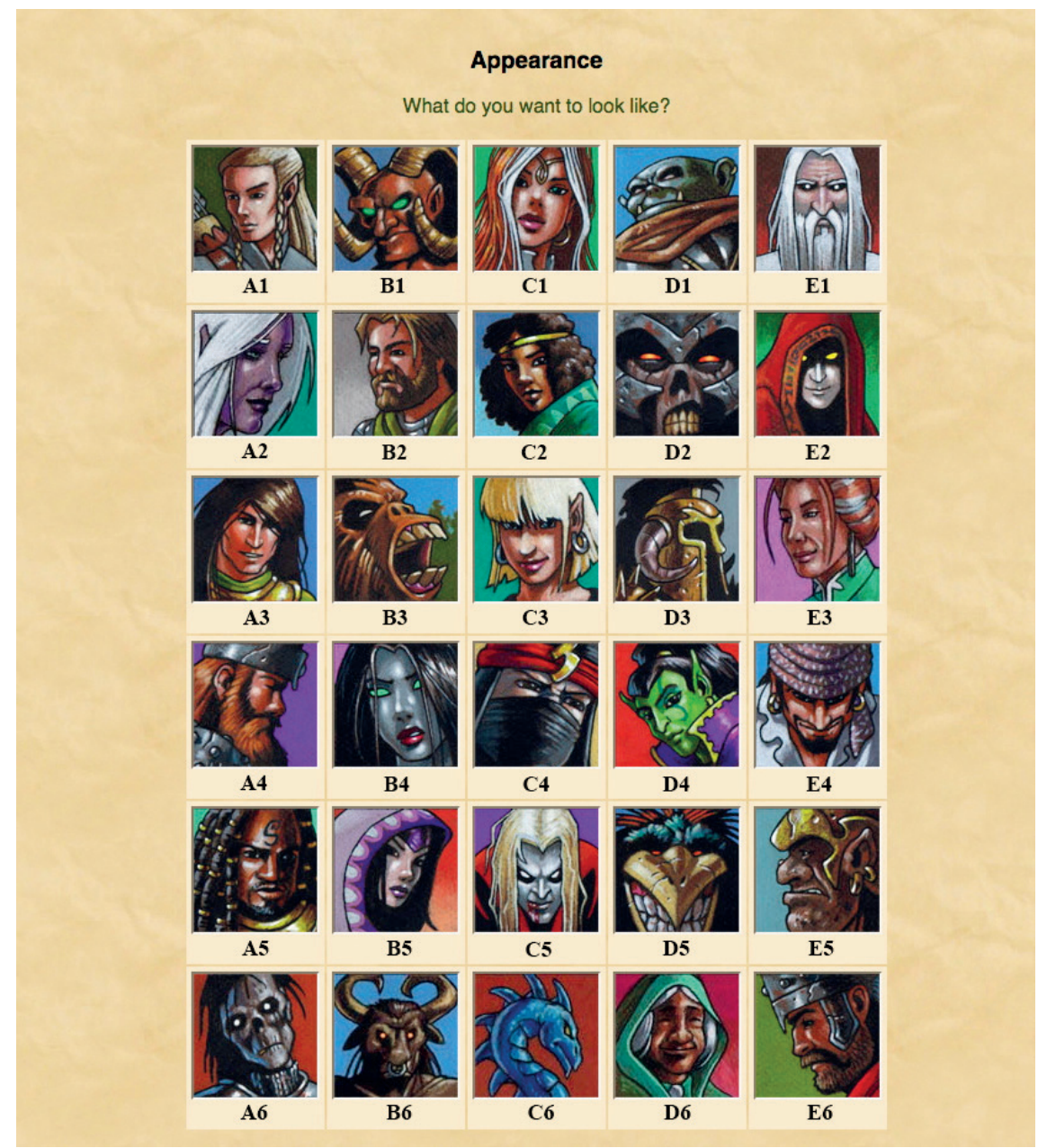

Ill. 2 - Galerie des avatars (capture d'écran)

Il sélectionne ensuite une série d'aptitudes (six au maximum) dans une liste prédéterminée.

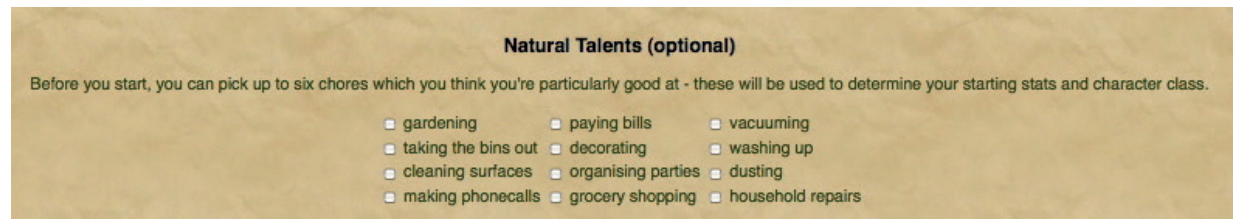

Ill. 3 - Liste des aptitudes (capture d'écran)

Ces deux opérations entraînent la création d'une fiche personnage: force (strenght), constitution (constitution), dextérité (dexterity), charisme (charisma), intelligence (intelligence), sagesse (wisdom). 
Les points sont attribués en fonction des aptitudes qui ont été déclarées. Il en va de même pour les rôles (maitre du donjon, homme de main [henchling], aventurier, apprenti), qui sont assortis de droits variés. Lors de la phase de jeu, les personnages évoluent. Ils ne peuvent pas mourir, mais ils peuvent changer de niveau et de classe d'appartenance. On peut s'engager dans une aventure en utilisant la liste de corvées prédéterminées proposée par le jeu ou en créant de toutes pièces sa propre liste.

\section{Liste des corvées prédéterminées}

\begin{tabular}{|c|c|c|c|c|}
\hline $\begin{array}{c}\text { THE } \\
\text { BATHROOM } \\
\end{array}$ & $\begin{array}{c}\text { THE } \\
\text { BEDROOM }\end{array}$ & $\begin{array}{c}\text { THE } \\
\text { KITCHEN }\end{array}$ & $\begin{array}{c}\text { THE OUTSIDE } \\
\text { WORLD }\end{array}$ & NO LOCATION \\
\hline $\begin{array}{l}\text { Cleaning the } \\
\text { bathroom }\end{array}$ & Making a bed & $\begin{array}{l}\text { Attending } \\
\text { to the } \\
\text { dishwasher } \\
\text { Making } \\
\text { dinner } \\
\text { Washing } \\
\text { dishes }\end{array}$ & $\begin{array}{l}\text { Gardening } \\
\text { Grocery shopping } \\
\text { Mowing the lawn } \\
\text { Taking out the } \\
\text { trash }\end{array}$ & $\begin{array}{l}\text { Doing the laundry } \\
\text { Dusting } \\
\text { Ironing } \\
\text { Paying the bills } \\
\text { Vacuuming } \\
\text { Watering plants }\end{array}$ \\
\hline
\end{tabular}

Le principe est déclaratif. Pour que le jeu considère que vous avez tondu la pelouse, il suffit de cocher la case adéquate. En cas de triche, le maître du jeu peut intervenir et rétablir la vérité. Chaque corvée est associée à des icones de couleur cuivre, or ou argent, permettant d'identifier les domaines de compétences qu'elle renforcera et le nombre de points qu'elle rapportera. Toute corvée déclarée effectuée permet de gagner de l'expérience, des pièces d'or et éventuellement des trésors. Ces gains sont fonction du temps nécessaire pour effectuer la tâche en question mais cependant modifiables pour adaptation à la pénibilité de la tâche (faire un lit en écoutant de la musique/nettoyer les sanitaires). En cas d'urgence (par exemple nettoyer la cuisine suite à une inondation due à une panne de machine à laver), il est possible de créer une quête. Aussi, bien sûr, il y a des combats, qui ont lieu lorsque le jeu le décide, souvent, quand deux personnages exécutent de façon plus ou moins simultanée une même tâche. Les adversaires sont des monstres. Le combat, pré-calculé, est présenté en mode texte. 


\section{Liste des monstres}

\begin{tabular}{|c|c|c|c|c|}
\hline THE & THE & THE & THE OUTSIDE & NO LOCATION \\
BATHROOM & BEDROOM & KITCHEN & WORLD & \\
\hline & & & Bladegrass patch & \\
& & & $\begin{array}{c}\text { Carnivorous plant } \\
\text { Cockroach }\end{array}$ & \\
& & & Enraged & Cactus beast \\
& & Fire & Dust bunny \\
& & shopkeeper & Dust golem \\
& & Fire sprite & Fire ant colony & Giant Rat \\
Drain beast & & Giant rat & Giant Spider \\
Giant spider & \multirow{3}{*}{ Bed bug } & Gelatinous & Mimic & Paper golem \\
Tentacle & Ghost & Red dragon & Moss beats & Soap elemental \\
Water & & Tentache & Paper golem & Steam elemental \\
elemental & & beast & Rat & Triffid \\
& & Water & Shopping Troll & Turker above \\
& & elemental & Squirrel & Water elemental \\
& & Water goblin & Tanglewine & Whipwine \\
& & & Triffid & Zombie \\
& & & Whipwine & \\
& & & Wood elemental & \\
& & & Zombie & \\
& & & &
\end{tabular}

Concernant enfin l'utilisation des ressources, il est possible de dépenser son pécule virtuel, d'abandonner ses trésors. Ce type d'action n'a aucune conséquence dans la partie qui se joue, mais le fait qu'il soit visible par l'ensemble des participants permet d'effectuer des "transactions " IRL ${ }^{15}$. La partie ne se termine que lorsque les joueurs le décident. Ils peuvent s'accorder préalablement sur sa durée, mais rien ne les empêche de changer d'avis en cours de route et de choisir de jouer les prolongations ou d'abandonner. L'accès au jeu est gratuit, mais il faut débourser $10 \$$ si l'on souhaite débloquer certaines fonctionnalités (statistiques, suivi d'une aventure sur une temporalité longue, etc.).

Si donc on se réfère à la définition proposée par Alvarez et Djaouti ${ }^{16}$, Chore Wars est bien un jeu sérieux, une " application informatique, dont l'intention initiale est de combiner, avec cohérence, à la fois des aspects sérieux (serious) tels, de manière non exhaustive et non exclusive, l'enseignement, l'apprentissage, la communication, ou encore l'information, avec des ressorts ludiques issus du jeu vidéo (game) ». De façon plus générale, il s’inscrit

16. Julian Alvarez, Damien Djaouti, Introduction au Serious Game, Paris, Questions théoriques, 2010. 
dans ce qu'on nomme la ludification (ou gamification), soit, selon Stello Bonhomme et Carole Talon-Hugon ${ }^{17}$, l'extension du domaine du jeu et le transfert de ses mécanismes dans des situations non ludiques dans le but de fidéliser une clientèle, d'encourager une activité, de rendre une tâche plus attractive ${ }^{18}$. Mathieu Triclot, dans le dernier chapitre de son ouvrage Philosophie des jeux vidéo, s'intéresse lui aussi à ce phénomène et en résume le principe en une phrase: "C'est la réalité elle-même qui devient un jeu" ${ }^{19}$." La plus inconditionnelle des supporters de la gamification est, sans aucun doute, la game designeuse Jane McGonigal. Constatant que des millions de gens éprouvent plus de satisfactions dans les jeux en ligne que dans la vraie vie, elle en déduit que notre " réalité est cassée ${ }^{20}$ " et explique que transformer le monde en jeu vidéo peut résoudre ce problème. Le sous-titre de son livre, Why Games Make us Better and How They Can Change the World ${ }^{21}$, est explicite. Pour elle, la gamification est la solution et Chore Wars est un modèle du genre. Mathieu Triclot critique cette position et interroge les possibles dérives de la gamification. Il rappelle, par exemple, comment la mairie de New York a testé un programme gamifié de gestion des populations pauvres et met en garde contre le "totalitarisme de poche ${ }^{22}$ ". Ailleurs, sur la toile, AssocFUREUR déconstruit, dans une analyse marxiste, le point de vue de McGonigal, arguant qu'elle ne cherche pas à provoquer véritablement du changement, mais plutôt à opérer un glissement dans notre perception du monde et qu'elle fait fi des réalités sociales et économiques dans lesquelles se débattent les personnes, avant de conclure que sa proposition consiste en fait à suggérer " aux gens de s'inonder l'esprit de suffisamment d'illusions pour qu'ils ressentent de l'amusement là où il n'y en a pas dans leur existence ${ }^{23} »$. Dans le cas du jeu qui nous occupe, cependant, le fait que l'enjeu économique soit

17. Stello Bonhomme, Carole Talon-Hugon, "Esthétique des jeux vidéo », Nouvelle Revue d'esthétique, 11, 2013/1, p. 5-10. DOI : 10.3917/nre.011.0005.

18. Un autre jeu existe sur I-phone, dont le thème est les tâches domestiques : Epic Win (Rexbox, SuperMono, 2010). Je l'ai écarté de mon étude, car si l'univers convoqué et le principe ludique sont globalement les mêmes que dans Chore Wars, il s'agit, en fait, d'une application de gestion du temps qui se joue seul et n'a que faire de la question du partage des tâches. Site officiel du jeu : http://www.rexbox.co.uk/epicwin/ index.html, consulté le $1^{\text {er }}$ septembre 2017.

19. Mathieu Triclot, Philosophie des jeux vidéo, Paris, Zones, 2011, p. 231.

20. Jane McGonigal, Reality is Brocken: Why Games Make us Better and How They Can Change the World, New York, Penguin, 2011.

21. "Pourquoi les jeux nous rendent meilleur et comment ils peuvent changer le monde. » (Traduction de l'auteure.)

22. Mathieu Triclot, Philosophie des jeux vidéo, op. cit., p. 235.

23. En ligne : http://www.gameblog.fr/blogs/assocfureur/page16, consulté le 19 juillet 2017.

Fanny Lignon, "Les corvées domestiques sont-elles solubles dans le jeu vidéo? », S. \& R., n 45, printemps 2018, p. 157-171. 
modeste, et aussi que les personnes sont libres de jouer ou non, atténue grandement la portée de ce type de critique. Selon Mathieu Triclot néanmoins, la plupart des dispositifs gamifiés nous dépossèdent, contrairement aux vrais jeux vidéo, "de notre puissance de décision, de notre capacité à agir sur le monde et ses cadres ${ }^{24}$ ». Le chercheur appelle ainsi de ses vœux l'avènement d'une autre forme de gamification.

Ne pourrait-on pas imaginer, avec ces mêmes dispositifs, des jeux qui soient vraiment des jeux, qui augmentent notre puissance d'agir collectivement sur le monde plutôt qu'ils ne le détruisent $t^{25}$ ?

\section{Identités de sexes et déconstruction des stéréotypes}

Dans ma contribution à l'ouvrage Former à l'égalité : défi pour une mixité véritable $e^{26}$, j'explique que les images, dans le double cadre éducatif que j'ai défini dans l'introduction de cet article, doivent être exploitées différemment selon qu'elles sont stéréotypées, contre-stéréotypées ou non-stéréotypées. Si donc on souhaite travailler sur Chore Wars en classe, il apparait indispensable de déterminer préalablement le type d'images que ce jeu met en œuvre.

Observons, pour commencer, l'ensemble des avatars proposés à l'adoption sur la page d'accueil. L'image se présente sous la forme d'une série de photos d'identités. Le codage couleur et l'attribution d'un chiffre et d'une lettre mappartiennent. Ils signalent la catégorie d'appartenance la plus probable des individus représentés : monstres (violet), personnages masculins (vert), féminins (orange), indéterminés (gris). Cette catégorisation, bien évidemment, peut être discutée. Elle se fonde sur des éléments visuels culturellement situés dont la présence induit, globalement, pour un occidental du $\mathrm{XXI}^{\mathrm{e}}$ siècle, l'appartenance à un sexe en particulier. Ainsi, $\mathrm{j}$ 'ai décidé, après un temps d'hésitation et en raison de la forme de sa mâchoire, de classer A1 parmi les personnages masculins. Pour A3 et C4, en revanche, s'ils sont porteurs, en quantité suffisante, de signes permettant de les ranger dans la catégorie des êtres humains, rien ne permet de les classer définitivement dans la catégorie

24. Mathieu Triclot, Philosophie des jeux vidéo, op. cit., p. 234.

25. Ibid.

26. Fanny Lignon, «Que faire avec les images » dans Annie Lechenet, Mireille Baurens, Isabelle Collet (dir.), Former à l'égalité : défi pour une mixité véritable, Paris, L'Harmattan, 2016, p. 105-118. 


\section{Appearance}

What do you want to look like?
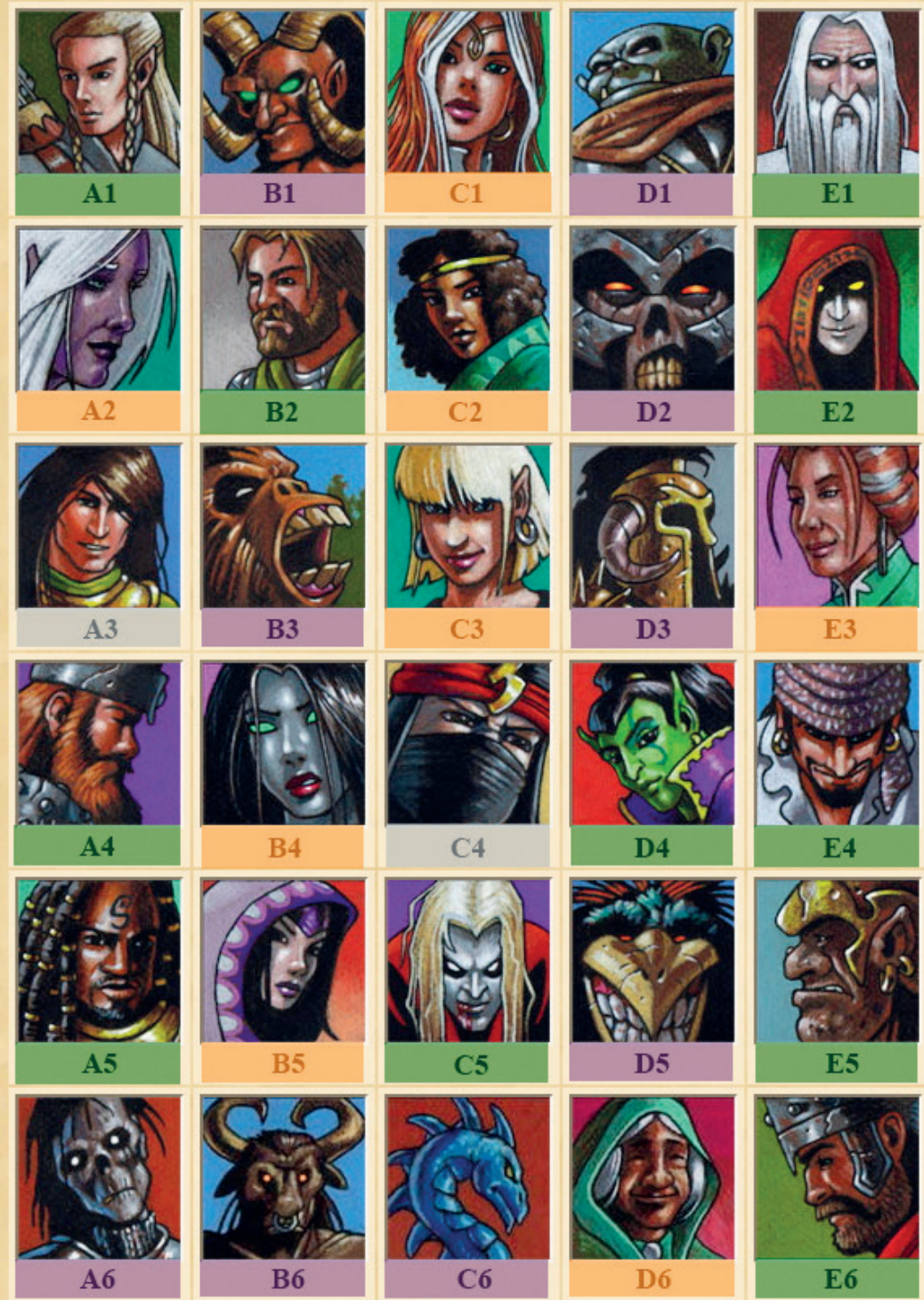

Ill. 5 - Galerie des avatars (capture d'écran avec application d'un codage couleur, d'une lettre et d'un chiffre) 
des hommes ou femmes. Le décompte auquel je suis arrivée est le suivant ${ }^{27}$ : onze hommes, huit femmes, deux personnages indéterminés, neuf monstres ${ }^{28}$. Le déséquilibre quantitatif entre les personnages féminins et masculins est à l'image de ce qu'on constate, de façon générale, dans les jeux vidéo ${ }^{29}$. Le thème bien particulier de Chore Wars invite cependant à l'interpréter comme un élément à part entière du discours, permettant peut-être de suggérer que les hommes pourraient en faire un peu plus. Aussi, le codage couleur met en lumière un autre élément d'importance. Que l'on considère, en effet, les lignes horizontales, les lignes verticales ou l'ensemble de la grille, les individus sont représentés pêle-mêle. Cette diversité est remarquable, car, dans la plupart des jeux, le choix du sexe est premier et ouvre l'accès à une liste de personnages jouables masculins ou féminins.

Regardons maintenant plus en détail les membres de ce groupe d'avatars. Notons pour commencer la variété des âges figurés. Cela va de jeune ${ }^{30}$ à vieux, en passant par des stades intermédiaires, pour les hommes (ex : E1) comme pour les femmes (ex : D6). Tous les personnages sont en gros plan. Ce choix esthético-technique, qui évite d'avoir à montrer les corps et les armes éventuelles, permet de présenter les personnes en tant qu'êtres pensants. Dans les images, les hommes sont très souvent de face quand les femmes sont plus souvent de 3/4 ou de profil, les regards aussi, souvent, étant genrés. Ici, cependant, tout est possible. Hommes, femmes et individus indéterminés sont présentés sous tous les angles. La variété est également de mise pour les regards. En A1 et A5, deux hommes baissent les yeux. En A3 et B4, deux femmes nous regardent droit dans les yeux. Si l'on s'intéresse à l'apparence et aux expressions, on constate que les personnages sont plus ou moins marqués

27. 11 hommes $(A 1+A 4+A 5+B 2+C 5+D 4+E 1+E 2+E 4+E 5+E 6) ; 8$ femmes $(A 2+B 4+B 5+C 1+$ $C 2+C 3+D 6+E 3) ; 2$ indéterminés (A3 et C4); 9 monstres (A6 + B1 + B3 + B6 + C6 + D1 + D2 + D3 + D5). 28. Après réflexion, j'ai choisi d'écarter les personnages non-humains de ma démonstration pour me focaliser sur le proche, le ressemblant. Si l'on considère les monstres (animaux, animaux fantastiques, démons, morts vivants), il est frappant cependant de constater que sur le plan du sexe, tous sont indéfinissables. Est-ce une façon de suggérer que l'indéterminé serait monstrueux? À cette question, je répondrai par la négative, pour plusieurs raisons. D’une part parce qu'il y a des individus indéterminés dans les rangs des humains aussi, d'autre part parce qu'il y a des éléments connotant le monstrueux (expressions démoniaques, regards jaunes et diaboliques) chez les humains hommes et femmes. Le rôle des monstres, ainsi, semble être d'offrir aux joueurs, aux joueuses, des possibilités autres d'incarner un personnage dont le sexe n'est pas défini.

29. Jesse Divnich, The Divnich Tapes: Female Representation in Games Across Genres, Consoles, Gamasutra, 22 octobre 2008. En ligne: http://www.gamasutra.com/php-bin/news_index.php?story=20771, consulté le 17 septembre 2017.

30. L'absence d'enfants s'explique aisément si l'on considère l'univers de référence, les MOORPG et le fait aussi que tout enfant aspire à devenir adulte.

Fanny Lignon, « Les corvées domestiques sont-elles solubles dans le jeu vidéo? », S. \& R., $\mathrm{n}^{\circ} 45$, printemps 2018, p. 157-171. 
sur le plan du sexe. L'éventail va du très masculin au très féminin avec, entre, toute une série de possibilités dont l'androgynie (A1, A3). Le sourire, dans les images médiatiques, est souvent l'apanage des femmes. Dans Chore Wars, les hommes aussi y ont droit (ex : B2). Inversement, l'agressivité et ses dérivés, qui sont généralement des attributs masculins, sont ici accessibles aux femmes (ex : B4). La question de la beauté se pose également. En règle générale, les productions de l'industrie vidéo-ludique mettent en scène de beaux humains et de belles humaines, la hideur étant plutôt réservée aux monstres. Ici, on remarque un homme laid en E5, mais aucune femme laide, comme si la laideur féminine était le dernier tabou. En ce qui concerne les vêtements et accessoires, on constate, là encore, une grande diversité. Les têtes sont indifféremment nues ou couvertes. Les coiffures sont variées. Les bijoux et autres ornements sont également répartis entre les hommes et les femmes. Enfin, on remarque que les bleus et les roses ne sont pas affectés à un sexe en particulier, mais sont distribués au même titre que toutes les autres couleurs (par exemple le rouge). Les fonds vont du vert au violet, pour les uns comme pour les unes. Les couleurs de peau oscillent, de façon plus ou moins réaliste, entre le rose et le vert en passant par le brun et le gris, les yeux sont bruns, jaunes, blancs ou vert vif.

La page d'accueil que je viens d'analyser reprend donc tous les codes, tant iconiques que plastiques, du masculin et du féminin ${ }^{31}$. Néanmoins :

- d'autres éléments sont présents qui disent le neutre ou ne sont porteurs d'aucune connotation genrée;

- l'accumulation sur un même individu est évitée;

- les éléments genrés, qu'ils disent le féminin ou le masculin, sont distribués équitablement entre les femmes, les hommes, les indéterminés, les monstres.

De cela il résulte que l'identification du sexe se fait, sauf exception, sans difficulté majeure alors que dans le même temps les stéréotypes sont déconstruits. Chore Wars démontre ainsi qu'il est possible de se passer des clichés sexistes et que cela n'affecte ni la qualité du jeu ni l'identité des personnages ${ }^{32}$.

31. Elle se réfère aussi, bien entendu et en tout premier lieu - il ne s'agit pas de l'oublier - aux MMORPG et aux personnages (guerriers, elfes, nains, mages, orques...) qui habitent les mondes de l'Heroic Fantasy. 32. À titre de comparaison, signalons qu'Epic Win ne propose que cinq avatars : un nain, une princesse guerrière, un guerrier, un squelette, un arbre. Sur le ton de l'humour, les hommes sont présentés comme des caricatures de virilité (corps "bodybuildé », air féroce et déterminé), les femmes comme des caricatures de féminité (armure ajourée, sourire, seins volumineux). 
Après analyse, nous pouvons donc dire que les images employées dans Chore Wars relèvent de la troisième catégorie, soit le non-stéréotype. On peut aussi, raisonnablement, en déduire la position de Kevan Davis sur la question du partage des tâches ménagères entre les hommes et les femmes, mais sans s'avancer trop cependant, puisque jamais il ne dit ouvertement qu'il s'agit d'un des objectifs de son jeu. Les représentations déployées vont même au-delà du non-stéréotype, en ce qu'elles rendent manifeste le travail de déconstruction des clichés. Elles illustrent ainsi parfaitement la notion d'anti-stéréotype qui, selon le sociologue Éric Macé :

[...] constitue, dans une logique contre-hégémonique, les stéréotypes comme la matière même de sa réflexivité, conduisant ainsi, en les rendant visibles, à déstabiliser les attendus essentialistes ${ }^{33}$.

\section{Conclusion}

La plupart des ouvrages ${ }^{34}$ qui dispensent aux enseignants des conseils pour aider leurs élèves à lutter contre les stéréotypes de sexe en images proposent des activités destinées à leur apprendre à les identifier et à les déconstruire. François de Singly explique cependant que cela ne saurait suffire.

Si les stéréotypes de la princesse et du soldat sont critiquables, il ne suffit pas d'affirmer (même si c'est juste!) le droit des petits garçons à être princes et des petites filles à être guerrières, il faut se demander comment un petit garçon peut s'affirmer en tant que petit garçon et une petite fille en tant que petite fille. $S^{\prime}$ il n'existe pas un nouvel imaginaire, alors il est plus que probable que les stéréotypes traditionnels perdurent ${ }^{35}$.

33. Éric Macé, "Des "minorités visibles" aux néostéréotypes », Journal des anthropologues, hors-série, 12 juin 2010. » En ligne : http://jda.revues.org/2967, consulté le 1er septembre 2017. DOI : 10.4000/jda.2967 34. Citons par exemple: Virginie Houadec, Michèle Babillot, 50 activités pour l'égalité filles-garçons à l'école, Toulouse, SCÉRÉN-CRDP, 2008; Sylvie Cromer, Danièle Hourbette, Suzanne Robichon, Guide des ressources pour les actions d'éducation à l'égalité filles garçons, Observatoire de l'égalité femmes-hommes, Secrétariat général de la ville de Paris, 2012 (en ligne : https://m.centre-hubertine-auclert.fr/outil/guidede-ressources-pour-les-actions-d-education-a-l-egalite-filles-garcons, consulté le 2 mars 2018); Virginie Houadec, Michèle Babillot, Astrid La Motte, Claire Pontais, 50 activités pour l'égalité filles-garçons, t. 1 : école primaire, 6e-SEGPA, Paris, Réseau Canopé, 2015.

35. François De Singly, Quels bons stéréotypes pour le genre et la famille?, LeMonde.fr., 2014. En ligne : http://www.lemonde.fr/idees/article/2014/02/07/quels-bons-stereotypes-pour-le-genre-et-la-famille_ 4361538_3232.html, consulté le 19 juillet 2017. 
Dans cette optique, une reconstruction est parfois envisagée, mais elle se contente, bien souvent, de jouer sur les contre-stéréotypes en inversant terme à terme les clichés. L'anti-stéréotype est souvent cité en exemple comme un idéal vers lequel il faut tendre. Toutefois, les images relevant de cette catégorie sont considérées comme assez peu courantes, difficiles à identifier, complexes à analyser et sont, en conséquence, rarement exploitées pédagogiquement, ce qui pose problème, car insinue qu'atteindre l'égalité $\mathrm{F} / \mathrm{H}$ est compliqué, voire impossible. L'objectif étant toutefois d' "éduquer à ", il serait sans doute doublement profitable, pour aider les élèves à concevoir et construire l'égalité en images, de les faire travailler sur des supports qui s'efforcent de se libérer vraiment des stéréotypes, comme ce jeu que je viens d'étudier.

Chore Wars place, en effet, l'égalité entre les femmes et les hommes au cœur du jeu. Sur le plan visuel, il nous propose un monde où cette égalité est réalisée, quand bien même elle est encore imparfaite, et suggère que, dans ces conditions, l'ennuyeux peut devenir amusant. Autrement dit, ce jeu nous explique que pour transformer des corvées en plaisir, il faut et il suffit que l'égalité entre les femmes et les hommes soit effective. Et il nous place dans cette situation idéale pour nous en montrer les avantages et avec pour ambition de faire bouger les choses. Cela en fait, en définitive, un jeu politique, dont le titre doit être pris au premier degré. Chore Wars ou Les guerres des corvées redéfinit de fait les sphères d'opposition, la guerre n'ayant plus lieu entre les individus, les femmes, les hommes, mais contre les corvées. Il se pose aussi en exemple de cette autre gamification espérée par Mathieu Triclot en ce qu'il propose, comme l'écrit ce dernier, " un usage positif et subversif de la gamification ${ }^{36}$ ". De cela je conclus que Chore Wars constitue un tremplin dont les enseignants pourraient s'emparer pour éduquer - comme en se jouant, sérieusement et simultanément - à l'image et à l'égalité entre les femmes et les hommes.

36. Mathieu Triclot, Philosophie des jeux vidéo, op. cit., p. 234.

Fanny Lignon, « Les corvées domestiques sont-elles solubles dans le jeu vidéo? », S. \& R., n 45, printemps 2018, p. 157-171. 